\title{
USING LIGHT TO SEE NEUTRONS AND ACCESSING THE 2D HIGH RESOLUTION: BAROTRON: A NEW PULSE FOR NEUTRON SCATTERING
}

\author{
Patrick Baroni and Laurence Noirez \\ Laboratoire Léon Brillouin (CEA-CNRS), CE-Saclay, 91191 Gif-sur-Yvette Cédex, France
}

Received 2014-05-28; Revised 2014-06-13; Accepted 2014-07-26

\begin{abstract}
Since first years of exploitation in 1960-70, neutron scattering has emerged as a unique and nondestructive means to probing inside matter properties at the nanometer length scales. Because the neutron production is scarce and expensive, the detection has to be extremely efficient. We take advantage of the recent improvements of light sensors in photon detection and detection dynamics, to develop a new type of two-dimensional neutron detector combining high detection efficiency and high spatial resolution. A prototype named Barotron (from the name of the inventor) has been built on this strategy. This 2D-neutron detector displays exceptional performances: Wide reciprocal space observation, spatial resolution lower than $0.5 \mathrm{~mm}$, low detection threshold $\left(<1\right.$ neutron $\left./ \mathrm{cm}^{2} / \mathrm{s}\right)$, reduced dimensions and a permanent possibility of upgrading. We underline the advantage of associating an accurate wavelength selection and point out the possibility to operate in time of flight mode. This type of instruments certainly foreshadows the future neutron scattering landscape, in particular in the view of future spallation sources.
\end{abstract}

Keywords: Neutron Scattering, High Resolution, Detection, Nanostructure

\section{INTRODUCTION}

Unlike X-Ray and Light Scattering, Neutron Scattering does not interact with electrons of atoms but is sensible to the nuclei. Because offering a unique contrast between elements as close hydrogen or deuterium, between atoms in metallic pieces or penetrating thick samples without damaging them, Neutron Scattering provides a unique probe to explore condensed matter at the nanometer length scales. Neutrons penetrate easily various materials compared to X-ray or electrons (which is advantageous when the sample is retained in a container), but correlatively weakly scatter. Compared to X-ray instruments at synchrotron sources with fluxes of $10^{18}$ photons $/ \mathrm{s} / \mathrm{mm}^{2}$, neutron fluxes delivered by continuous sources is weak (at maximum with $10^{4}$ neutrons $/ \mathrm{s} / \mathrm{mm}^{2}$ ) (Anderson et al., 2009). Inventing highly efficient neutron detectors is thus of prime importance for an improved approach of the structural and dynamic properties of matter.

In the current state of the art, neutron scattering at 2 dimensions was typically developed for Small Angle Neutron Scattering (SANS), i.e., adapted for the characterization of nanostructures (polymer, colloids, aggregates, clays, membranes, lipids, DNA ...). The conventional ${ }^{3} \mathrm{He}$ or $\mathrm{BF}_{3}$ gas-chamber Position-Sensitive Detectors (gas-PSD) devoted to SANS have a low spectral resolution because of the several millimeters size of the elementary cells of the detector. The gas detector operates via the detection of an ionized particle resulting from the neutron absorption by the gas, those free electrons are collected on a high voltage electronic Corresponding Author: Laurence Noirez, Laboratoire Léon Brillouin (CEA-CNRS), CE-Saclay, 91191 Gif-sur-Yvette Cédex, France 
grid. The technology of the position sensitive gaschamber detectors can hardly evolve since the spatial resolution is limited by the large pixel size (several millimeters side) and by their number scarcely flexible because of electronic limitations (Belushkin, 2008). On the other hand, the $\mathrm{Gd}$ image-plate detectors initially designed for the X-rays radiation and adapted for neutron detection present a high spatial resolution but are limited by a slow photoemission $(0.1 \mathrm{~s})$, a long relaxation time $(0.9 \mathrm{~s})$, a sensitivity to gamma radiations, memory effects (due to the activation of Baryum) and gamma production (Anderson and McGreevy, 2009; Cipriani et al., 1994). Their performances are generally insufficient to access to a weak signal/noise rate required to measure low contrast factors. Defining a new generation of position-sensitive neutron detector specifically dedicated to neutron scattering is thus an urgent need.

Initiated about a couple of years ago, the project called "Barotron" (from one of its inventor names) offers a novel mean to explore the elastic neutron scattering domain combining a high spatial resolution, an efficient detection rate, an access to a broad scattering space and an easy use. These objectives are neither satisfyingly fulfilled by gas-detectors nor by image-plate systems. The new two-dimensional position sensitive detector couples the advantages of a solid scintillator in terms of excellent spatial resolution to the evolution of the new charge-coupled detection devices (Baroni et al., 2006). This solid-PSD uses a coupling of a photoemission means (LiFZnS solid scintillator) adapted for the neutron radiation, a high performance cooled Charge-Coupled Detection (CCD) camera system and a light amplifier. This setup is different from ICCD (intensified couple charge devices) and enables a full well capacity of at least 65000 e-/pixel, accessing to a true 16 bits dynamics which is inaccessible with ICCD. The LiFZnS solid scintillator is chosen for its very low rate of detection of gamma, its high and fast photoconversion rate of neutrons (about $10^{5}$ photons/neutron) and its fast relaxation time (about 200ns). The resulting twodimensional neutron detector displays a large and versatile detection area $(260 \times 260 \mathrm{~mm})$, a high spatial resolution $(250000$ pixels of $0.35 \times 0.35 \mathrm{~mm}$ ), a very low detection threshold $\left(<1\right.$ neutron $/ \mathrm{cm}^{2} / \mathrm{s}$-determined by absolute calibration via ${ }^{3} \mathrm{He}$ chamber), a true 16 bits dynamic range that ensures a linear intensity measurement, data viewable in situ during the acquisition and a sensitivity equivalent to gas-detector with respect to the standard neutron wavelength range from $0.5 \AA$ to $20 \AA$ (Fig. 1); i.e., detection performances competing with the best gas chamber detectors with additionally a spatial resolution comparable to X-Ray or synchrotron radiation 2D-detectors. The combination of these technical advances enables a detailed and quantitative observation of the reciprocal space. The high spatial resolution extends the possibilities to use reduced size samples which is a non-negligible advantage when the material is rare or difficult to synthesize (labelled samples). Finally, the compact size of this new neutron detector allows a high versatility including the use in neutronography mode (neutron imaging).

The present paper updates the best performances reachable with this neutron detector, points out the interest of the high resolution even for the study of soft matter or low organized materials and shows the advantages of narrowing the resolution in wavelength distribution on examples at small and at large scattering angles.

\section{MATERIALS AND METHOD}

\subsection{Selecting Neutron Wavelength}

There are two principal modes of selection of neutron wavelength: The first mean is a mechanical selector (inset of Fig. 2a) (Rosta, 1989). It selects the wavelength according to the neutron velocity.

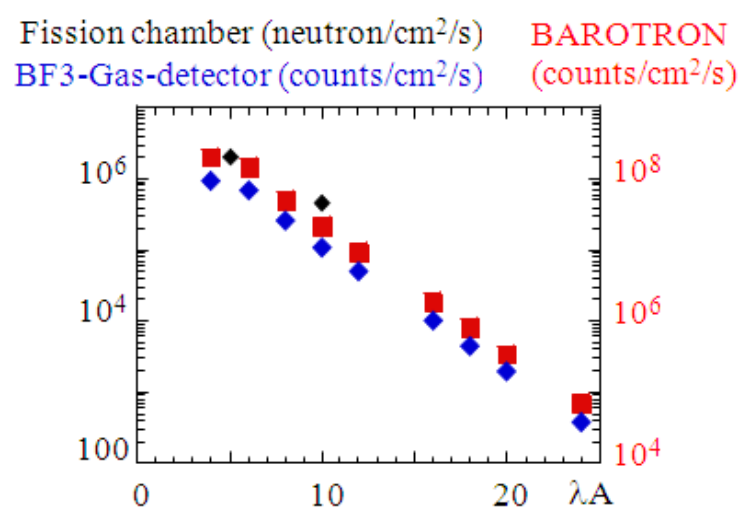

Fig.1. Comparison of the efficiencies of different types of neutron detection with respect to the wavelength. Integrated intensity (acquisition time: 10s); $\diamond$ : BF3-Gas-detector (PAXY) counts $/ \mathrm{cm}^{2} / \mathrm{s}$; $\diamond$ : Fission chamber (absolute calibration): Neutrons $/ \mathrm{cm}^{2} / \mathrm{s} ; \mathbf{\square}$ : CDD-detector Barotron (Li scintillator), counts $/ \mathrm{cm}^{2} / \mathrm{s}$ 
This $h$ one $v^{2}$ follows the de Broglie formula:

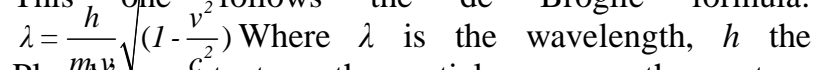
Planck's constant, $m$ the particle mass, $v$ the neutron velocity and $c$ the celerity of light in vacuum. The instrument consists in a cylinder with helical vacuum openings alternating with a Gadolinium neutron absorber (inset of Fig. 2a). The velocity v, the step P and the frequency $\omega$ are linked by the equation: $\mathrm{V} \sim P$. $\omega / 2 \pi$ (Rosta, 1989). The wavelength band-width $(\Delta \lambda / \lambda)$ is about $10-15 \%$. The low resolution of the mechanical selector produces typically an anisotropic stretching of the scattered beam which is all the more important that the scattering vector is large. The mechanical selector offers a wide flexibility in the choice of the wavelength (usually ranged from $3 \AA$ up to $20 \AA$ ). Being aligned along the neutron guide, it also delivers a substantial flux of neutrons which represents about $10 \%$ of the incident white beam. This type of mechanical selector equips typically gaschamber spectrometers. Because of the several millimeters size of their elementary cells (typically from $5 \mathrm{~mm}$ up to $10 \mathrm{~mm}$ pixel side size), these detectors have a low spatial resolution. These spectrometers are typically devoted for the study of soft matter or of weakly organized matter and/or to scattering at small angles for which the resolution is not of primary importance (the more the scattering vector $Q$ small is, the smaller the uncertainty $\Delta Q$ ) while taking advantage of a significant neutron flux.

The second means to select the wavelength is a crystalline monochromator (inset of Fig. 1b). It exploits the laws of reflection on aligned monocrystals. For a given angle, only one wavelength is allowed according to the law of Bragg $(2 \theta=2 \arcsin (\lambda / 2 d))$. The bandwith on the wavelength depends on the mosaicity of the monochromator. It remains very weak, lower than $\Delta \lambda / \lambda<$ $2 \%$. The wavelength is very well defined but the reflected beam is less than $2 \%$ of the incident white beam (Currat, 1973). This type of monochromator is typically associated to elastic and inelastic neutron spectrometers demanding a high wavelength resolution for the fine characterization of crystalline structures or of an accurate energy transfer determination.

\section{RESULTS}

\subsection{Wide Scattering Angles and Resolution Effects}

We first illustrate the effect of resolution at wide scattering angles examining the neutron pattern displayed by a stretched semi-crystalline polymer (polyethylene). The sample is fully deuterated to lower the incoherent background noise. The stretching produces a reorientation of the micro-crystallites along the axis of stretching in agreement with previous X-Rays observations (Murthy and Minor, 1990; Aggarwal and Tilley, 1995; Ohde et al., 1971). Several characteristic distances are present of which the distance between polymer chains indexed by an orthorhombic packing with lattice dimensions $a=7.4$ $\AA, b=4.94 \AA$ and $c=2.54 \AA$. The experiment consists in observing by diffraction the same reflections (110) and (200) of the orthorhombic packing of the polyethylene sample oriented by cold drawing) on a neutron guide equipped by either a mechanical selector and a conventional gas-position sensitive detector, PAXY of the Lab. Léon Brillouin, or a graphite monochromator coupled with the high resolution detector Barotron. The incident polychromatic beam and the collimation are identical and the wavelength chosen to be the same $(\lambda=3.6 \AA)$. The sample-detector distance is calculated to typically focus the observation on the same reflection peak corresponding to the lattice dimensions $b=4.94 \AA$ ( $Q$ $\left.=1.2 \AA^{-1}\right)$. Figure $\mathbf{2 a}$ displays the neutron pattern produced by the conventional $\left(\mathrm{BF}_{3}\right)$ gas positionsensitive detector (PAXY) equipped with a mechanical selector $(\Delta \lambda / \lambda=13-15 \%)$. The detector is placed at $850 \mathrm{~mm}$ from the sample and rotated by an angle of $20^{\circ}$ with respect to the incident beam to locate the reflection peak at $1.2 \AA^{-1}$ on the detector area. The detector displays one reflection peak at $Q=$ $1.2 \AA^{-1}$ with an apparent mosaïcity along the direction of the scattering vector of $0.33 \AA^{-1}$ (FWHM). Figure 2b displays the neutron pattern measured with the high resolution detector Barotron coupled to a graphite monochromator $(\Delta \lambda / \lambda$ less than $2 \%)$. The detector is placed at $60 \mathrm{~mm}$ from the sample. In this configuration, the detector plane accesses the full scattering cone from $Q=0$ up to $Q=2.14 \AA^{-1}$. In addition, the neutron pattern (Fig. 2b) reveals two reflection peaks (100) and (200) along the direction perpendicular to the stretching of the Polyethylene at $\mathrm{Q}=1.2 \AA^{-1}$ and at $Q=1.3 \AA^{-1}$ respectively. The weaker intensity reflection located at $Q=1.3 \AA^{-1}$ is related to the helical shape of the molecule (Murthy and Minor, 1990; Ohde et al., 1971). This weak reflection is unidentifiable with a high wavelength dispersion (Fig. 2a) since it merges with the high intensity reflection at $Q=1.2 \AA^{-1}$. The width of each 
reflection does not exceed $0.15 \AA^{-1}$ (FWHM corrected from instrumental resolution), i.e., about twice narrower than the one produced with a mechanical selector. The degradation of the resolution produced by a mechanical selector occurs along the radial directions only, the resolution along the transverse direction to the scattering vector being unaffected. The widths of the reflections along the transverse direction, in the two configurations corresponding to Fig. 2a and $\mathbf{b}$ indicate indeed a similar value (FWHM $=0.31-0.32 \AA^{-1}$, corrected from instrumental resolution). The anisotropy of the wavelength dispersion originates from the neutron velocity dispersion which gives raise to Bragg reflections at slightly different radial positions on the detector.

\subsection{Structure Resolved at Small Scattering Angles}

The second example concerns soft matter organization. Various materials exhibit lamellae phases of several nanometers interlayer distance (liquid crystals, micellar solutions, colloidal suspensions, copolymers, clays, membranes, lipids, DNA...). An interlayer order $30 \AA$ or more is a frequent value, corresponding to a scattering visible at $0.2 \AA^{-1}$ in the so-called Small Angle region (SANS). We show here that reducing the wavelength dispersion at small angles is of interest even for weakly ordered materials. We examine and compare the twodimensional scattering produced by the same sample viewed by the conventional gas-detector equipped with a mechanical selector (Fig. 3a) and by the high resolution detector equipped a graphite monochromator (Fig. 3b). The sample is a liquid crystal monodomain previously aligned with a magnetic field (Noirez et al., 1995; Noirez, 2000). The patterns (Fig. 3a and 3b) display a Bragg reflection at $\mathrm{q}=0.20 \AA^{-1}$ along the horizontal indicating the average direction of the normal to the lamellae. The width along the transverse direction defines the crystal mosaicity whereas the range of the lamellae order is defined by the width along the longitudinal direction. The mosaicity obtained using the conventional gas-detector equipped with the mechanical selector (Fig. 3a), is about $0.015 \AA^{-1}$. The mosaicity is of the same order $\left(0.0158 \AA^{-1}\right)$ using the high resolution Barotron with a graphite monochromatic (Fig. 2b). In contrast, the conventional detector equipped with the mechanical selector indicates a longitudinal width of about $0.027 \AA^{-1}$ which corresponds to the limit of resolution whereas the high resolution Barotron with a graphite monochromatic, indicates that the width along the longitudinal axis is of about $0.016 \AA^{-1}$ (which is also the limit for this instrumental resolution). The wavelength dispersion induces at small angles a significant widening of the shape of the reflection (rate of 1.7 at $\mathrm{q}=0.2 \AA^{-1}$ ) with as a consequence an underestimation of the range of the correlations along the longitudinal axis of the scattering vector when a mechanical selector is used.

\subsection{Small Angle Neutron Scattering}

In a third example, we compare the efficiency of the Barotron versus conventional gas-detector. Figure 4a and 4b display the small angle neutron patterns produced by the same sample using identical scattering conditions (identical wavelength, collimation, incident flux and acquisition time) and changing the two detection means only. The first spectrum is recorded on a conventional detector (PAXY) and the second one is recorded on the new device (Barotron). This experiment has been carried out by positioning the Barotron which is of reduced dimensions, in front of the conventional detector. This strategy guaranties the strict use of identical experimental conditions. The comparison of the two patterns (Fig. 4a and 4b) illustrates the interest of exploring the reciprocal space using a large array of pixels and a high counting level (the gas-chamber detector and the solid detector Barotron count an electronic discharge and a photon emission respectively).

This advantage is particularly visible at short acquisition time since the high number of pixels compensates the intensity value dispersion and thus improves the statistic together with the counting of large numbers of photons that improves the precision. Finally, position-sensitive gas detectors use a time to digital converter; i.e., alternating an elementary detection of the neutron (identified by its potential) and a readout dead-time. The use of CCD system allows the simultaneous counting of all the events on the detector and therefore is not penalized by event by event detection principle. This series of characteristics advantages the new Barotron system particularly interesting in case of kinetic experiments. 


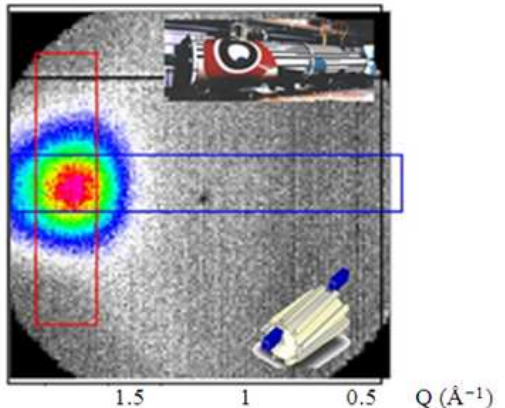

(a)

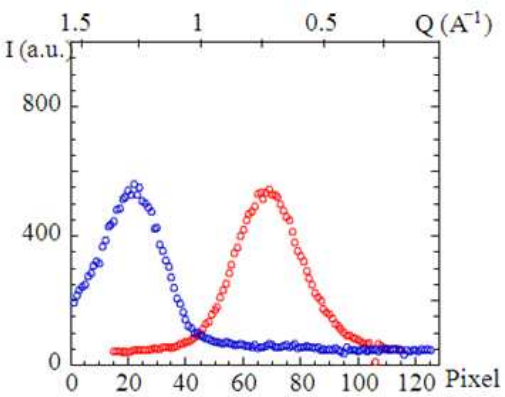

(b)

Fig. 2a. 2D neutron pattern recorded on a conventional $128 \times 128$ pixels BF3 detector PAXY, $7 \mathrm{~m}$ long, 5 tons (photo at the top inset) equipped with a mechanical selector (scheme in bottom inset) and corresponding profiles along $(O)$ and transverse $(O)$ to the reflection. The sample is a deuterated semi-crystalline polyethylene (stretched vertically to a rate $1 / 10=7$ ). Sampledetector distance: $850 \mathrm{~mm}, \lambda=3 \AA$, wavelength dispersion $\Delta \lambda / \lambda \approx 13 \%$, $4 \mathrm{~mm}$ diaphragm, acquisition time: $900 \mathrm{~s}$, Incident flux: $5.10^{6}$ Neutrons $/ \mathrm{cm}^{2} / \mathrm{s}$

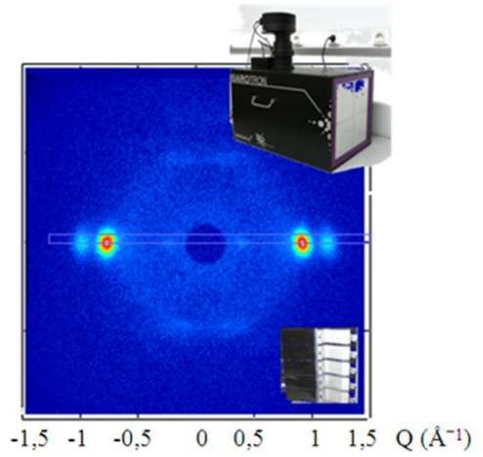

(a)

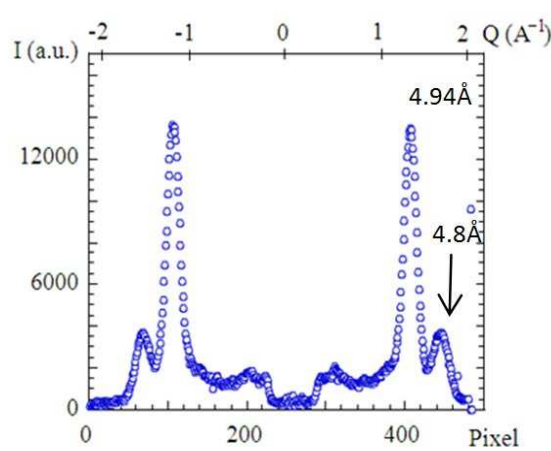

(b)

Fig. 2b. Same sample: 2D pattern (without correction, smoothing or binning) of wide angle neutron spectrum viewed with the new Barotron detector, $0.5 \mathrm{~m}$ long, $20 \mathrm{~kg}$ (inset picture at the top) equipped with a graphite monochromator (inset picture at the bottom) (sample-detector distance: $60 \mathrm{~mm}, \lambda=3.6 \AA$, $4 \mathrm{~mm}$ diaphragm, wavelength dispersion $\Delta \lambda / \lambda \approx 2 \%$, acquisition time: 900 s (incident flux: $6.10^{5}$ neutrons $/ \mathrm{cm}^{2} / \mathrm{s}$ )) and the corresponding profile (O)along the 110 and 002 reflections.

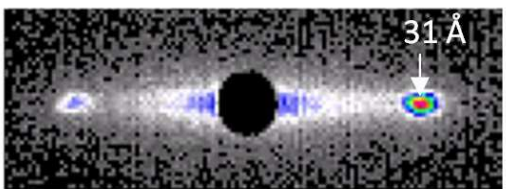

(a)

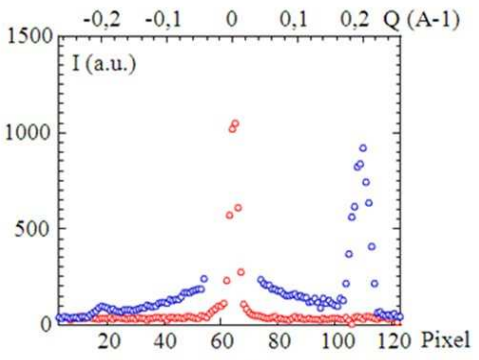

(b)

Fig. 3a. Conventional BF3 detector $(128 \times 128$ pixels $)$ equipped with a mechanical selector $(\lambda=6 \AA, \Delta \lambda / \lambda=13 \%$, sampledetector distance $=1.2 \mathrm{~m})$ : SANS pattern and profiles along $(O)$ and transverse $(O)$ to the reflection pattern of a lamellar phase (bottom figure). The Bragg reflection is extended along the horizontal axis due to the dispersion of the wavelength. (The central scattering is related to the chain form factor related to the polymer chain onto which the liquid crystal moieties are attached (Noirez et al., 1995) 


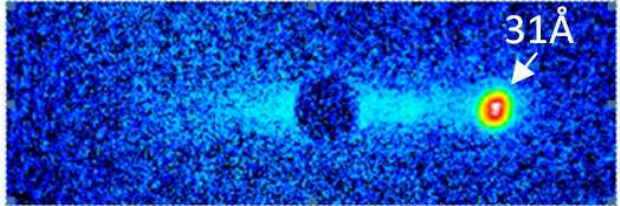

(a)

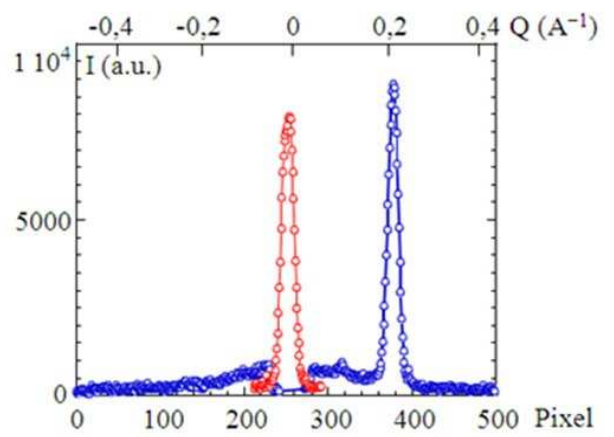

(b)

Fig. 3b. Barotron detector $(512 \times 512$ pixels $)$ with graphite monochromator: SANS pattern of the same sample (lamellar phase) $(\lambda=$ $3.6 \AA, \Delta \lambda / \lambda=2 \%$, sample-detector distance $=0.31 \mathrm{~m}$ ) and profiles along $(\mathrm{O})$ and transverse $(\mathrm{O})$ to the reflection (bottom figure). The shape of the Bragg reflection is restored and well-defined (half of the detector data are displayed)

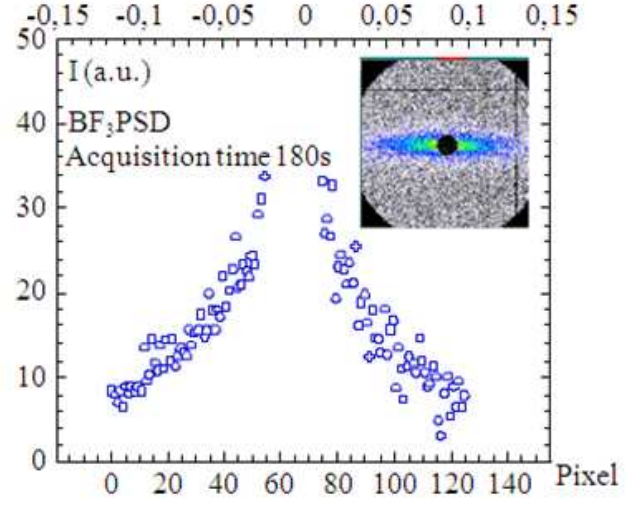

(a)

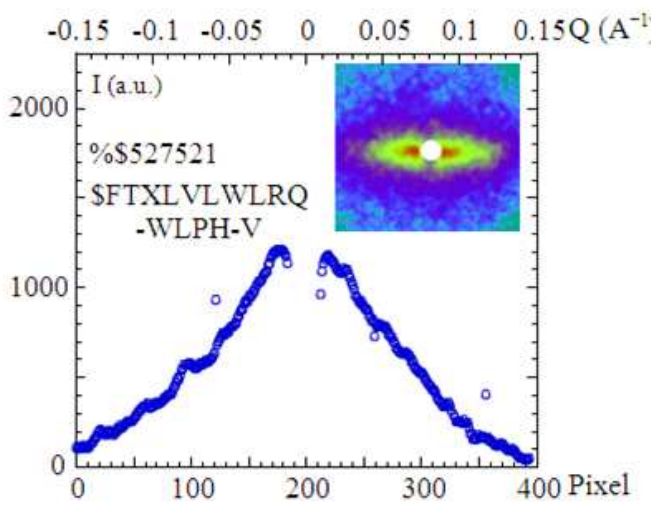

(b)

Fig. 4. Comparison of the spectra (horizontal cut) recorded by the two detection means: conventional BF3 PSD (PAXY) and solid detector (BAROTRON) corresponding to left and right figures respectively. The patterns are recorded by simply exchanging the two detectors (acquisition time: 180s, collimation: 10mm, wavelength: $\lambda=10 \AA$ ), using the same sample (hydrogenated/deuterated mixture of oriented polymer chains) and identical scattering conditions.

\section{CONCLUSION}

To conclude, high resolution 2-dimensional measurements open new horizons for probing organized matter from low to wide scattering angles (typically up to $5 \AA^{-1}$ ). Up to now, intermediate and wide angle scattering were rarely explored and exploited in soft matter for both small and large molecules. For example, the determination of the spatial distribution of the intermolecular interactions can be crucial to understand how these materials react when they are submitted to anisotropic internal or external fields as stretching (Casas et al., 2008), flow, magnetic or electric field as already applied in the floating waterbridge experiment (Fuchs et al., 2010)... This range is well adapted to the mesoscopic sizes. The example of room temperature ionic liquids (RTILs) (Judeinstein et al., 2013) reveals that some of them form organised phases apt to orient in a low magnetic field (Fig. 5). This type of study remains hardly accessible using conventional PSD detectors. Applications in the neutronography mode (neutron imaging) are also accessible: Fig. 6 describes a neutron-imaging kinetics experiment of infiltration of a $3 \mathrm{~mm}$ sphere via the modification of the transmittances of the solvent and of the sphere. The spatial resolution reaches $20 \mu \mathrm{m}$. Various neutron imaging applications can profit of the high penetration length of the neutron beam particularly in metal or stone studies or when the samples are thick or X-Ray absorber. Neutrons are irreplaceable and complementary to X-Ray imaging. 

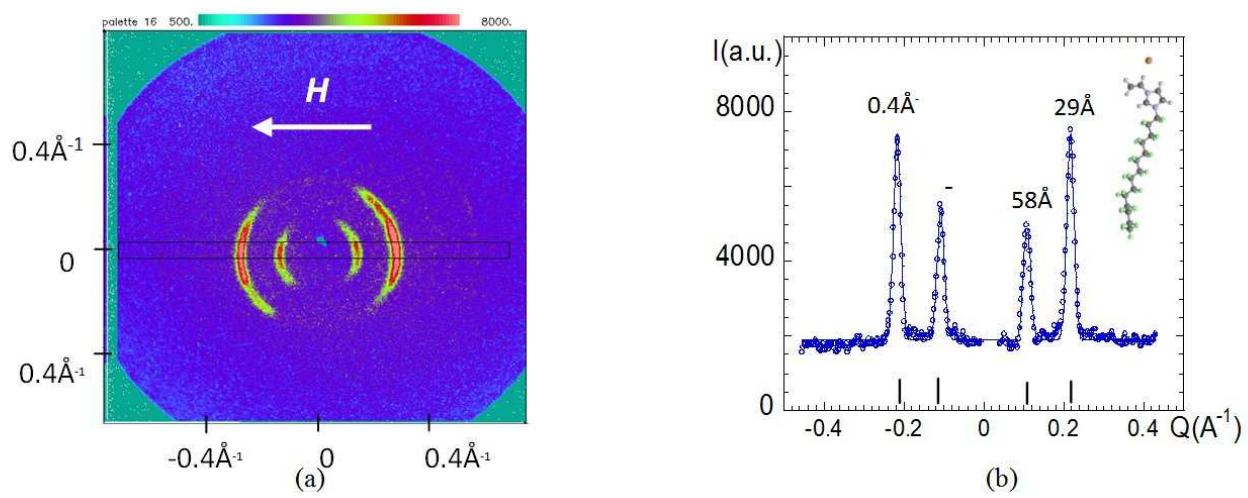

(b)

Fig. 5. 2D-neutron pattern and horizontal cut recorded on Barotron detector enlightening the structure of a Room Temperature Ionic Liquid (RTIL). The layered organization and the ability of the RTIL to align along a magnetic field (horizontal direction) are evidenced by the azimuthal distribution of the intensity centered along the magnetic field direction. Raw 2D pattern obtained without correction, smoothing or binning. With the courtesy of P. Judeinstein.

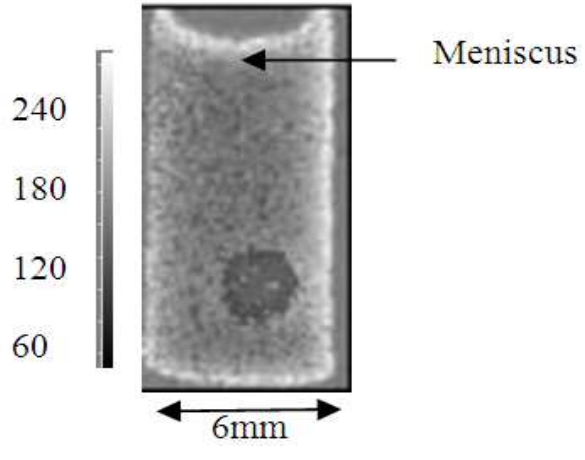

Fig. 6. Acquisition in Neutron-imaging mode of the kinetics of infiltration of a solvent in a porous mineral sphere of $3 \mathrm{~mm}$ diameter (capillary tube $6 \mathrm{~mm}$ ). Neutron transmission picture obtained using the portable device, spatial resolution $20 \mu \mathrm{m}$ (Babyrotron)

As long as neutrons will provide unique information about the structure of materials accessible with none other technique, innovating in neutron detection is an utmost challenge to compensate their weak interactions with the matter and the low fluxes. The recent advances in photon detection and detection dynamics of the CCD cameras make them extremely attractive and evolutionary as new neutron scattering detection means when they are coupled to a neutron scintillator. This new type of PSDs based on solid scintillators coupled to low level CCD reaches unequally spatial resolution in matter of neutron scattering, accesses to very low detection threshold ( $<1$ neutron $/ \mathrm{cm}^{2} / \mathrm{s}$ ) and to wide scattering angles. High number of pixels and large counting rates specific to the photon detection improve advantageously the dynamic and the statistic which is of interest particularly for kinetic experiments. With reduced dimensions, this new type of PSD combines ideally the advantages of X-ray devices in terms of spatial resolution and wide observation plane, to the unique properties of neutron scattering. Its detection efficiency competes now with the best gas chamber detectors and can be used as well for the observation from large scattering angles to small scattering angles. The application cases illustrated here highlight the pertinence of using a monochromatic neutron beam when the spatial resolution of the detector is high. The example at wide angles (stretched polyethylene) illustrates typically the loss of information from using a $10 \%$ dispersed wavelength and which produces the melt of two distinct Bragg reflections separated by a few degrees in wider one. The comparison of the three application cases points out also the irrelevance at broadening the size of conventional gasdetector to access comparable reciprocal space. About nine conventional gas-detectors are required to cover a cone of diffusion equivalent to that displayed by the Barotron. Additionally an equivalent resolution is reachable only if the huge detector is associated to a high resolution monochromator which is clearly unrealistic. In conclusion, PSDs based on solid scintillators coupled to low level CCD as the Barotron will certainly assume significant proportions in the future of the neutron scattering landscape. In addition to undeniable performances, its versatility enables various applications including neutron imaging (illustrated here 
by the liquid migration in microporous systems) or kinetic scattering measurements. This ergonomic device exhibits for a reasonable financial investment, high performances that revisit the way of building neutron detection means. In the context of further optimization of the performances, the constant evolution of the sensors enables a permanent upgrade, a customization of the dynamic range, light and radiation sensitivities, noises and speed parameters following the needs of the experiment. Finally, the development of new fast speed reading sensors, accessing times of flight below the millisecond while keeping high dynamics and a low level detection threshold, open for the first time the perspectives of application of CCD detectors to inelastic measurements (analysis in terms of energy). This possible evolution is clearly of prime interest, especially in the frame of the developments of the future spallation source.

In these new challenges, gathering both scientific and instrumental expertises is crucial in order to define and repel the parameters limiting the performances.

\section{ACKNOWLEDGEMENT}

The authors are grateful to A. Laverdunt, G. Exil for the "Pingouin" interface, G. Pépy for the 2-dimensional software "HWXY" (available on request) and P. Monceau (Néel Institut) for his valuable support.

\section{REFERENCES}

Aggarwal, S.L. and G. P. Tilley, 1955. Determination of crystallinity in polyethylene by X-ray diffractometer. J. Polymer Sci., 18: 17-26. DOI: 10.1002/pol.1955.120188702

Anderson, I.S. and R. McGreevy, 2009. Neutron Imaging and Applications: A reference for the Imaging Community (Neutron Scattering Applications and Techniques). 1st Edn., Springer, ISBN-10: 0387786929, pp: 400.

Anderson, I.S., A.J. Hurd and R. McGreevy, 2009. Neutron Scattering Applications and Techniques.

Baroni, P. and L. Noirez, 2006. Two-dimensional detection system for neutron radiation in the field of neutron scattering spectrometry. PCT WO2006/095013 A.

Belushkin, A.V., 2008. Modern trends in the development of position sensitive neutron detectors for condensed matter research. Pramana, 71: 639651. DOI: 10.1007/s12043-008-0252-4
Casas, F., C. Alba-Simionesco, H. Montes and F. Lequeux, 2008. Length-scale of glassy polymer plastic flow: A neutron scattering study. 41: 860860. DOI: $10.1021 / \mathrm{ma} 0714969$

Cipriani, F., F. Dauvergne, A. Gabriel, C. Wilkinson and M.S. Lehmann, 1994. Image plate detectors for macromolecular neutron diffractometry, Biophys. Chem., 53: 5-13. DOI: 10.1016/03014622(94)00071-9

Currat, R., 1973. The efficiency of vertically bent neutron monochromators. Nuclear Instruments Methods, 107: 21-28. DOI: 10.1016/0029554X(73)90007-4

Fuchs, E.C., P. Baroni, B. Bitschnau and L. Noirez, 2010. Two-dimensional neutron scattering in a floating heavy water bridge. J. Phys. D: Appled Phys., 43: 105502-105502. DOI: 10.1088/00223727/43/10/105502

Judeinstein, P., S. Huet and P. Lesot, 2013. Multiscale NMR investigation of mesogenic ionic-liquid electrolytes with strong anisotropic orientational and diffusional behavior. RSC Adv. 3: 16604-16611. DOI: 10.1039/C3RA00025G

Murthy, N. S. and H. Minor, 1990. General procedure for evaluating amorphous scattering and crystallinity from X-ray diffraction scans of semicrystalline polymers. Polymer, 31: 996-1002. DOI: 10.1016/0032-3861(90)90243-R

Noirez, L., 2000. Shear induced smectic-A-smectic-C transition in side-chain liquid-crystalline polymers. Phys. Rev. Lett., 84: 2164-2167. PMID: 11017234

Noirez, L., P. Keller and J.P. Cotton, 1995. On the structure and the chain conformation of side-chain liquid crystal polymers. Liq. Cryst., 18: 129-148. DOI: $10.1080 / 02678299508036602$

Ohde, Y., H. Miyaji and K. Asai, 1971. Deformation of sedimented mats of polyethylene single crystals, Japasene J. Applied Phys., 10: 171-171. DOI: 10.1143/JJAP.10.171

Pépy, G., L. Noirez, P. Keller, M. Lambert, F. Moussa, J.P. Cotton and H. Richard, 1999. Observation of the conformation and structure of some liquid-crystal polymers by small-angle neutron scattering. Die Makromolekulare Chemie, 191: 1383-1392. DOI: 10.1002/macp.1990.021910617

Rosta, L., 1989. Neutron physical properties of a multidisc velocity selector. Physica B: Condensed Matter, 156: 615-618. DOI: 10.1016/09214526(89)90747-3 